\title{
Graphoepitaxy of High-Quality GaN Layers on Graphene/6H-SiC
}

\author{
András Kovács, * Martial Duchamp, Rafal E. Dunin-Borkowski, Rositza Yakimova, \\ Péter L. Neumann, Hannes Behmenburg, Bartosz Foltynski, Cristoph Giesen, \\ Michael Heuken, and Béla Pécz**
}

The implementation of graphene layers in gallium nitride ( $\mathrm{GaN})$ heterostructure growth can solve self-heating problems in nitride-based high-power electronic and light-emitting optoelectronic devices. In the present study, high-quality GaN layers are grown on patterned graphene layers and $6 \mathrm{H}-\mathrm{SiC}$ by metalorganic chemical vapor deposition. A periodic pattern of graphene layers is fabricated on $6 \mathrm{H}-\mathrm{SiC}$ by using polymethyl methacrylate deposition and electron beam lithography, followed by etching using an $\mathrm{Ar} / \mathrm{O}_{2}$ gas atmosphere. Prior to $\mathrm{GaN}$ growth, an AIN buffer layer and an $\mathrm{Al}_{0.2} \mathrm{Ga}_{0.8} \mathrm{~N}$ transition layer are deposited. The atomic structures of the interfaces between the $6 \mathrm{H}-\mathrm{SiC}$ and graphene, as well as between the graphene and AIN, are studied using scanning transmission electron microscopy. Phase separation of the $\mathrm{Al}_{0.2} \mathrm{Ga}_{0.8} \mathrm{~N}$ transition layer into an AIN and GaN superlattice is observed. Above the continuous graphene layers, polycrystalline defective $\mathrm{GaN}$ is rapidly overgrown by better quality single-crystalline $\mathrm{GaN}$ from the etched regions. The lateral overgrowth of $\mathrm{GaN}$ results in the presence of a low density of dislocations $\left(\approx 10^{9} \mathrm{~cm}^{-2}\right)$ and inversion domains and the formation of a smooth GaN surface.

\section{Introduction}

Graphene has attracted tremendous attention over the past few years because of its 2D atomic structure and unique physical

Dr. A. Kovács, Dr. M. Duchamp,

Prof. R. E. Dunin-Borkowski

Ernst Ruska-Centre for Microscopy and Spectroscopy

with Electrons and Peter Grünberg Institute

Forschungszentrum Jülich

52425 Jülich, Germany

E-mail: a.kovacs@fz-juelich.de

Prof. R. Yakimova

Department of Physics

Chemistry and Biology

Linköping University

581 83, Linköping, Sweden

P. L. Neumann, Prof. B. Pécz

Institute for Technical Physics and Materials Science

Research Centre for Natural Sciences

Hungarian Academy of Sciences

P.O. Box 49, H-1525, Budapest, Hungary

E-mail: pecz@mfa.kfki.hu

Dr. H. Behmenburg, B. Foltynski, Dr. C. Giesen, Prof. M. Heuken AIXTRON SE

Kaiserstrasse 98, 52134, Herzogenrath, Germany

DOI: 10.1002/admi.201400230 properties, ${ }^{[1]}$ which are highly desirable for numerous applications. Graphene has a very high heat conductivityl[2] of up to $5000 \mathrm{~W} \mathrm{mK} \mathrm{m}^{-1}$, suggesting that it may be a suitable candidate for solving selfheating problems in nitride-based (e.g., $\mathrm{GaN}, \mathrm{InGaN}$, and AlGaN) high-power electronics $^{[3]}$ and solid state optoelectronics. ${ }^{[4]}$ High-quality epitaxial nitride layers can be grown on single-crystalline substrates, such as $\mathrm{ZnO}, \mathrm{SiC}$, and $\mathrm{Al}_{2} \mathrm{O}_{3}$ (sapphire), using intermediate buffer and transition layers. The lack of chemical reactivity between GaN (or AlN as a buffer) and graphene leads to imperfect polycrystalline growth, resulting in a large number of undesired defects in the $\mathrm{GaN}$ and a possible rough surface, making the integration of graphene into nitride thin film heterostructures very challenging. In order to overcome the growth issue, Han and co-workers ${ }^{[5]}$ used lithographically patterned graphene oxide to improve heat dissipation in light-emitting diodes (LEDs). Chung et $\mathrm{al}^{\left[{ }^{[6]}\right.}$ used a $\mathrm{ZnO}$ coating on $\mathrm{O}_{2}$ plasma-treated graphene layers to grow high-quality $\mathrm{GaN}$ layers and to make the resulting heterostructure transferable to any substrate. Yan et al. ${ }^{[7]}$ implemented thermal heat-escaping channels from graphene layers on top of AlGaN/GaN transistors deposited on SiC.

In the present study, we show that a $c$-axis-oriented GaN layer that has high structural perfection can be grown by metalorganic chemical vapor deposition on patterned graphene layers and (0001) oriented $6 \mathrm{H}-\mathrm{SiC}$ using an AlN buffer and $\mathrm{Al}_{0.2} \mathrm{Ga}_{0.8} \mathrm{~N}$ transition layers. The nucleation and growth of the heterostructure are studied using advanced transmission electron microscopy (TEM) in cross-sectional geometry. The GaN layer is observed to have a smooth surface and an estimated dislocation density of $3 \times 10^{9} \mathrm{~cm}^{-2}$, which is in the same range as the dislocation density in a control sample grown without graphene layers. We show that the $\mathrm{GaN}$ that is formed above the continuous graphene is polycrystalline and is overgrown laterally by single-crystalline GaN.

\section{Results and Discussion}

\subsection{Etching of Graphene and Nitride Deposition}

Graphene layers were prepared on the Si-terminated face of an 0001-oriented 6H-SiC single crystal 2 in. wafer by 
a high-temperature sublimation process $^{[8]}$ developed at Linköping University. Auger electron spectroscopy, ${ }^{[9]}$ was used to determine that the number of graphene layers was usually $2-3$, with small regions containing up to ten graphene layers. The continuous graphene layers were covered with polymethyl methacrylate (PMMA) and patterned using electron beam lithography, to leave $1 \mu \mathrm{m}$ wide unmasked areas in a $3 \mu \mathrm{m}$ periodic pattern, as shown in Figure 1a. The unmasked graphene layers were removed by high-density plasma etching using an Ar $(89 \%)$ and $\mathrm{O}_{2}(11 \%)$ mixture. Ar ions enhanced the etching mechanism by introducing defects into the graphene to create starting points for oxidation. ${ }^{[10,11]}$ The PMMA mask was dissolved using acetone. A $100 \mathrm{~nm}$ thick AlN buffer layer was then deposited onto the patterned graphene $/ 6 \mathrm{H}-\mathrm{SiC}$ surface, followed by the deposition of $\approx 300 \mathrm{~nm}$ thick $\mathrm{Al}_{0.2} \mathrm{Ga}_{0.8} \mathrm{~N}$ and $\approx 1.5 \mu \mathrm{m}$ thick GaN layers, as shown in Figure 1b.

\subsection{Microstructure and Crystallographic Orientation Relationship}

A preliminary visual check of the sample surface revealed a periodic pattern of lines, indicating irregular nitride growth. Figure 1c shows a scanning electron microscopy (SEM) image of the surface of the GaN. The dark spots, which are inversion domains in the GaN, are present in lines of $1 \mu \mathrm{m}$ width and $3 \mu \mathrm{m}$ spacing. By comparing the dimensions of the inversion domain regions with the geometry of the graphene patterning and etching, it is evident that the smooth $\mathrm{GaN}$ regions without inversion domains form above the graphene layers. However, as shown below, the etching process does not remove the graphene layers from the $\mathrm{SiC}$ perfectly, but leaves islands. The AlN grains then start to grow directly on the $\mathrm{SiC}$ and overgrow the graphene islands. Based on the SEM image shown in Figure 1c, the inversion domain density is determined to be $3 \times 10^{8} \mathrm{~cm}^{-2}$.

In order to study the growth of the heterostructure, the sample was analyzed in cross-sectional geometry. Figure $1 \mathrm{~d}$ shows a low-magnification bright-field (BF) TEM image of a cross-sectional sample of the heterostructure. The GaN layer can be seen to contain semicircular polycrystalline regions above the intact graphene layers. In Figure $1 \mathrm{~d}$, the straight vertical dark lines are inversion domains, which travel straight to the surface from regions where the AlN/GaN grows directly on the SiC. By estimating the specimen thickness using electron energy-loss spectroscopy (EELS) to be between 80 and $120 \mathrm{~nm}$ for the region shown in Figure 1d, the dislocation density was determined to be $\approx 3 \times 10^{9} \mathrm{~cm}^{-2}$. A control sample without graphene layers (see Supporting Information) was grown
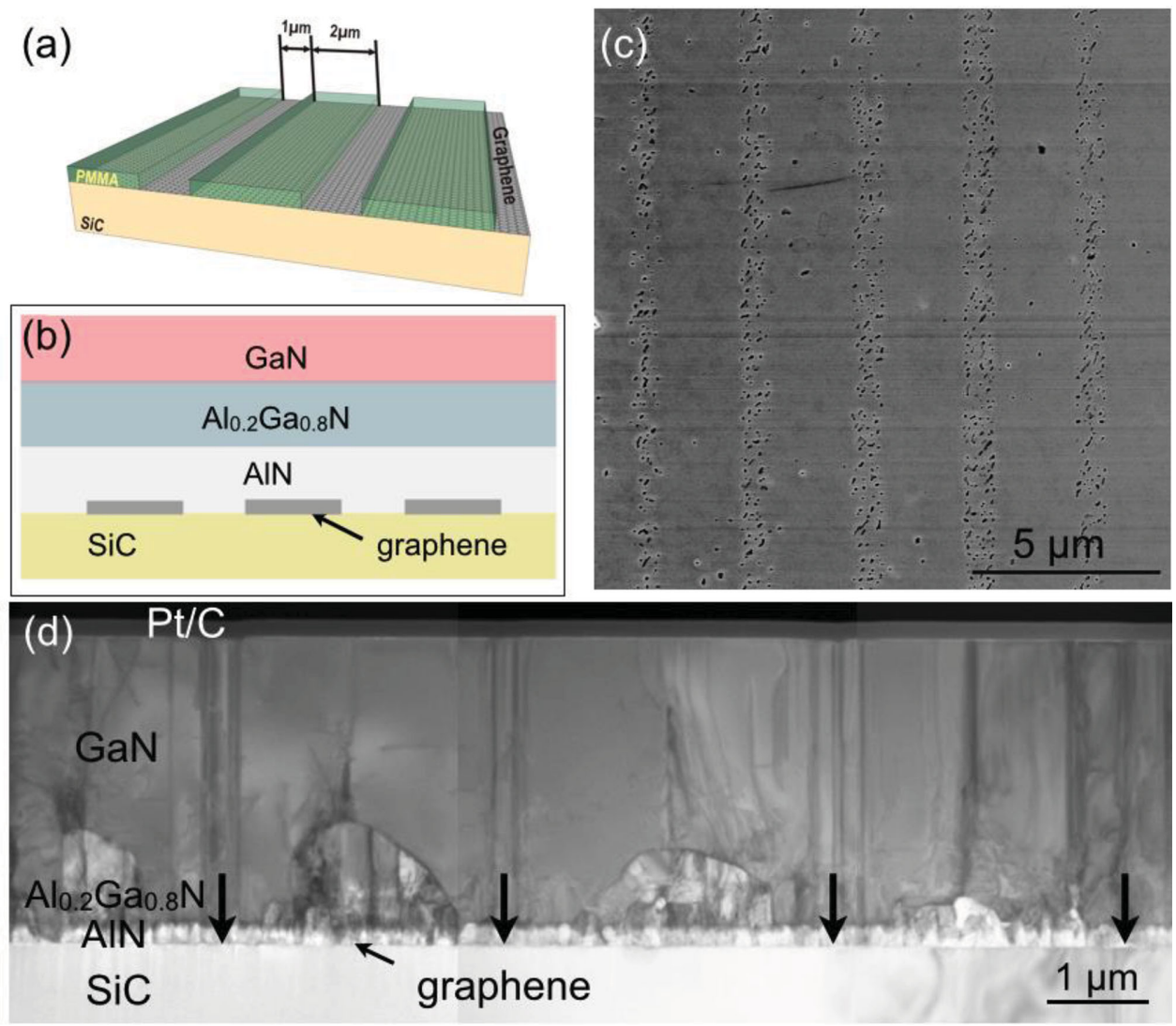

Figure 1. a) Schematic illustration of graphene layers patterned using PMMA. b) Schematic diagram showing the deposited layer sequence. c) SEM secondary electron image of the surface of the final heterostructure grown on the patterned graphene layers. The dark spots are inversion domains in the GaN layer. d) Low-magnification bright-field TEM image of the heterostructure. Arrows mark the regions where the graphene layers were partially etched away in $1 \mu \mathrm{m}$ wide regions. The $\mathrm{Pt} / \mathrm{C}$ is a protective layer deposited during specimen preparation to prevent $\mathrm{Ga-ion}$ implantation. The specimen thickness is $\approx 100 \mathrm{~nm}$. 

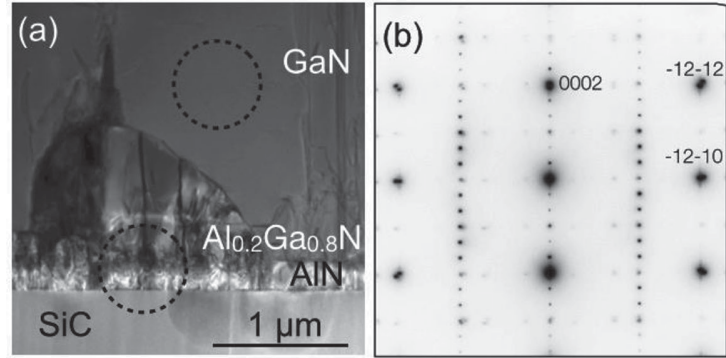

(c)

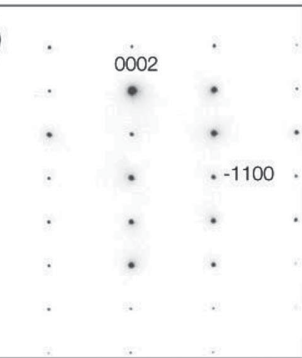

Figure 2. a) BF TEM image showing the transition region close to the substrate, where singlecrystalline $\mathrm{GaN}$ laterally overgrows the defective $\mathrm{GaN}$ that forms above the graphene layers. The dashed circles in (a) indicate the regions that were used to record SAED patterns from b) the substrate, $\mathrm{AIN}, \mathrm{Al}_{0.2} \mathrm{Ga}_{0.8} \mathrm{~N}$ and defective $\mathrm{GaN}$ and c) the single-crystalline $\mathrm{GaN}$.

using identical parameters and had a dislocation density of $2 \times 10^{9} \mathrm{~cm}^{-2}$.

The cross-sectional view shown in Figure 1d reveals halfcircle-shaped defective regions in the GaN, in which many grain boundaries are present. The microstructure of $\mathrm{GaN}$ around this defective region was studied using BF TEM images and selected area electron diffraction (SAED) patterns, as shown in Figure 2. The SAED pattern shown in Figure $2 \mathrm{~b}$ was recorded from a region that overlapped the substrate and the defective region containing the AlN and GaN layers, as marked by the lower circle in Figure 2a. The AlN on the graphene layers is epitaxial with the $\mathrm{SiC}$ substrate, according to the relationship $[11-20]_{\mathrm{AlN}}(0002)_{\mathrm{AIN}} / /[10-10]_{\mathrm{SiC}}(0002)_{\mathrm{SiC}}$. The splitting of the [-12-12] reflection in Figure $2 \mathrm{~b}$ results from the epitaxial growth of GaN on AlN. Interestingly, the GaN layer above the defective region has a [10-10] orientation, which is different from the [11-20] orientation observed outside the defective region. The overgrown $\mathrm{GaN}$ layer is, therefore, rotated by $30^{\circ}$ relative to the defective region.

\subsection{Chemically Sensitive Imaging of the Interfaces}

Compositionally sensitive high-angle annular dark-field (HAADF) scanning TEM (STEM) revealed the formation of a superlattice of AlN and GaN layers in the $300 \mathrm{~nm}$ thick $\mathrm{Al}_{0.2} \mathrm{Ga}_{0.8} \mathrm{~N}$, as shown in Figure 3, in which the contrast variations reflect the atomic number differences between $\mathrm{Al}(Z=$ $13)$ and $\mathrm{Ga}(Z=31)$ in the nitride layers. Figure 3a shows an HAADF STEM image recorded from a region close to the substrate. The AlN surface is rough, with grain height differences of between 50 and $200 \mathrm{~nm}$. The arrows indicate superlattice regions, which grow $\approx 500 \mathrm{~nm}$ into the GaN layer. The superlattice regions begin on the $\mathrm{AlN}$ grains where an $\mathrm{Al}_{0.2} \mathrm{Ga}_{0.8} \mathrm{~N}$ layer was supposed to grow according to the growth procedure. Figure 3b,c show magnified images of the AlN/GaN superlattices. Close to the GaN layer, the GaN layer thickness in the superlattice decreases from $\approx 10$ to $\approx 4 \mathrm{~nm}$. The high-resolution HAADF STEM image of the superlattice shown in Figure 3c was acquired from a region where the AlN is approximately four monolayers thick. The AlN and GaN layers are in perfect epitaxy, with no evidence of planar defects. Mohseni et al. ${ }^{[12]}$ observed phase separation in InGaAs nanowires grown on graphene and described it as a special case of van der Waals epitaxy. In our experiments, the $\mathrm{Al}_{02} \mathrm{Ga}_{0.8} \mathrm{~N}$ layer did not grow directly on the graphene layers and so the phase separation cannot be explained in the same way. The superlattice formation mechanism is not understood at present, but is thought to be related to the growth of the nitride layers on graphene. Although $\mathrm{C}$ contamination could inhibit phase pure $\mathrm{Al}_{0.2} \mathrm{Ga}_{0.8} \mathrm{~N}$ layer growth, highresolution compositional experiments using energy-dispersive X-ray spectroscopy and EELS were not able to reveal $\mathrm{C}$ enrichment at the interfaces between the AlN and GaN layers. However, the measurement of small C concentration differences is very challenging using TEM, especially as the high-energy electron beam can deposit $\mathrm{C}$ onto the surface of the specimen. In order to further elucidate the mechanism of AlN/GaN superlattice formation, a control sample grown without graphene layers using an identical procedure was also studied (see Supporting Information). An AlN/GaN superlattice was not observed in the latter sample, suggesting that the phase separation may indeed originate from the presence of $\mathrm{C}$ during layer growth.

The growth of AlN grains on intact and etched graphene layers was also studied using high-resolution HAADF STEM. Figure 4 shows HAADF STEM and BF STEM images recorded simultaneously from the interfacial regions, providing information about both the formation of graphene layers on $6 \mathrm{H}-\mathrm{SiC}$
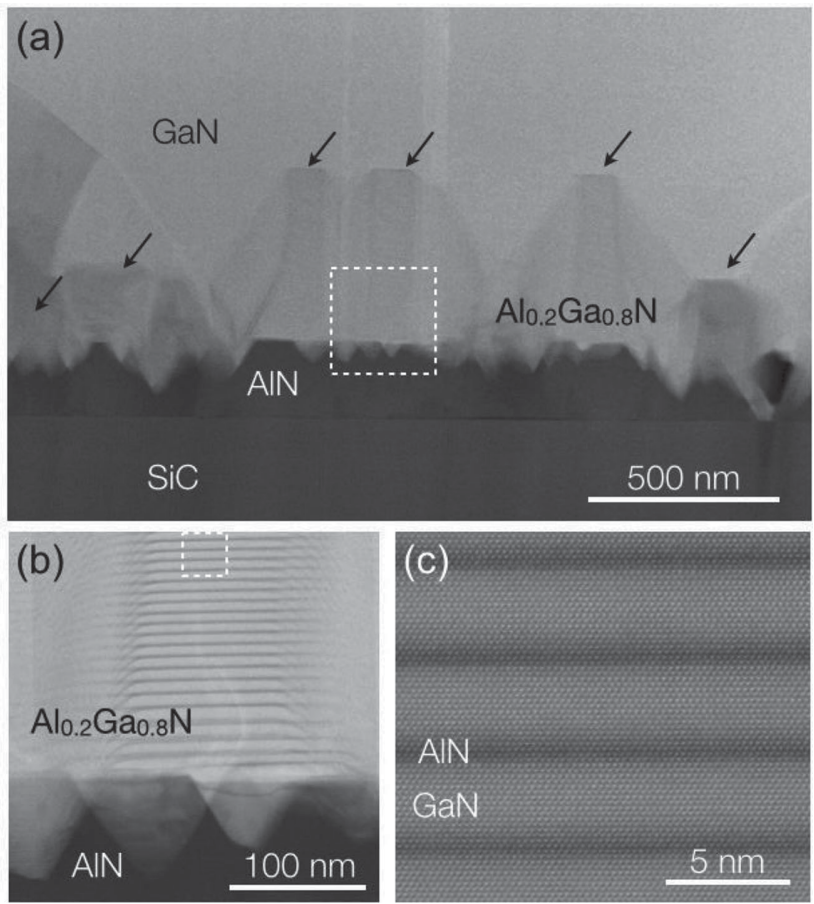

Figure 3. a) STEM HAADF images of the AIN/GaN superlattices, which are marked with arrows. The inner ADF detector semiangle used was $78 \mathrm{mrad}$. The dashed rectangles in (a) and in (b) indicate the regions shown in (b) and (c), respectively. In (b), the AIN and GaN layer periodicity decreases in the growth direction. The atomic-resolution HAADF STEM image in (c) shows sharp interfaces, with an AIN layer thickness of $\approx 1 \mathrm{~nm}$. 


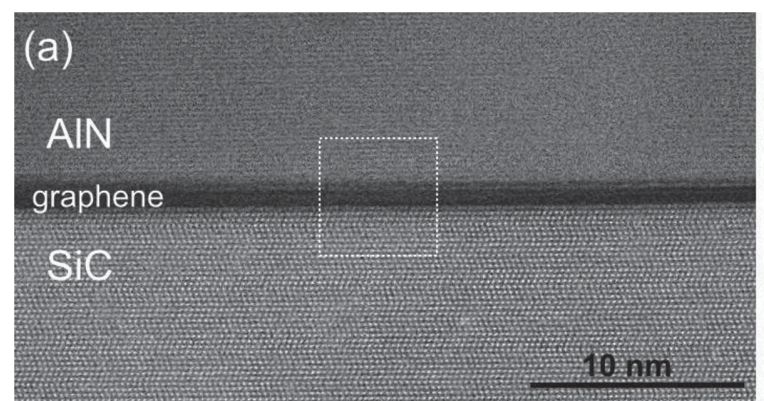

(c)
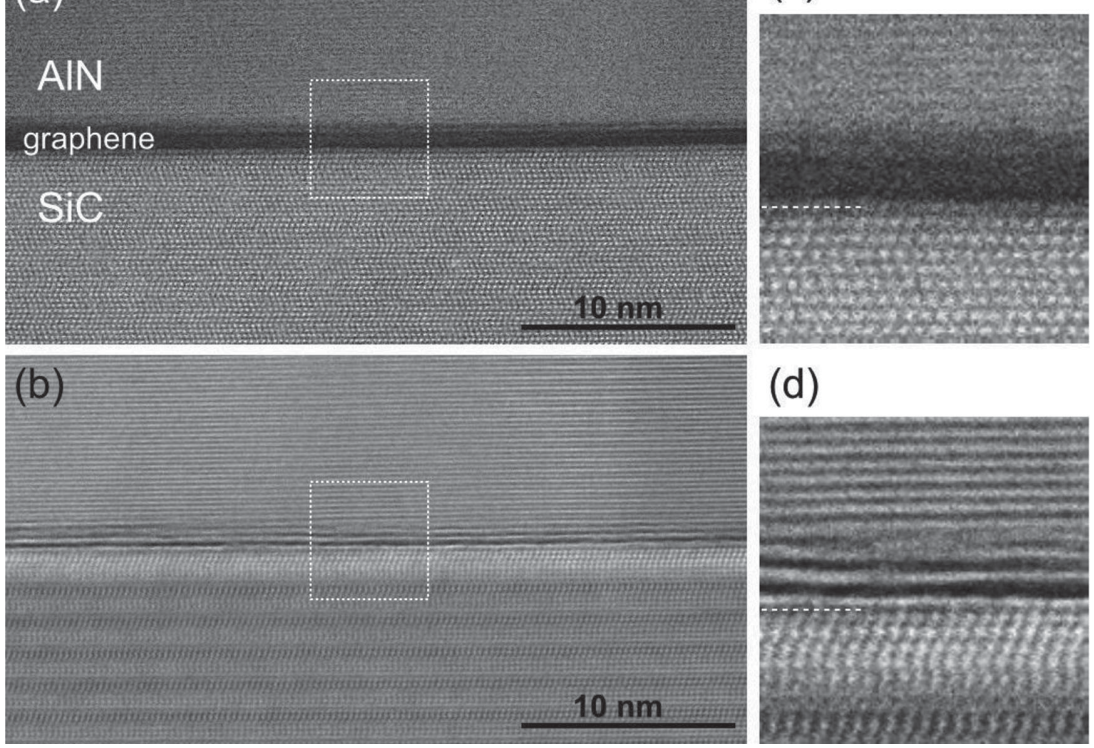

(d)

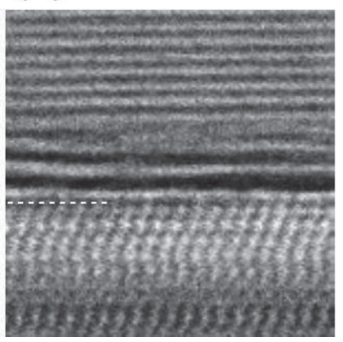

Figure 4. Atomic-resolution a) HAADF STEM and b) BF STEM images of AIN grown on nonetched graphene layers. The regions marked by dashed rectangles in (a) and (b) are enlarged in (c) and (d), respectively. The graphene layers, Al and Si columns appear bright in the HAADF STEM images and dark in the BF STEM images. The uppermost SiC layer is marked by a dotted line. The inner ADF detector semi-angle used was $78 \mathrm{mrad}$.

and the growth of AlN on graphene. The AlN grain is observed to grow on three graphene layers. The image intensity of HAADF STEM, I depends from atomic number, $\approx Z^{1,7}$ that results in a lower contrast of carbon than of $\mathrm{Al}$ and $\mathrm{Si}$ atomic columns. In order to find the location of the graphene layers, BF STEM image is also shown in Figure $4 \mathrm{~b}$. The origin of the bright contrast in the uppermost $\mathrm{SiC}$ layers visible in Figure $4 \mathrm{~b}$ is not understood at present, but may be associated with compositional variations or strain. Yu et al.[13] showed that the presence of strain at a $\mathrm{SiO}_{2} /$ $\mathrm{Si}$ interface results in a change in ADF STEM image intensity, which depends on inner detector semiangle and specimen thickness. However, the high-temperature annealing of $6 \mathrm{H}-\mathrm{SiC}$ that was used to prepare the graphene layers may also have led to a slightly off-stoichiometric composition in the last few layers of the $6 \mathrm{H}-\mathrm{SiC}$, with $\mathrm{Si}$ diffusing out. The sharp interface between the AlN grain and the graphene layers shows that the PMMA layer was removed completely and that the AlN grew directly on the graphene.

As described above, $\mathrm{Ar} / \mathrm{O}_{2}$ plasma etching was used to remove the graphene layers in stripes from the $\mathrm{SiC}$ surface. Figure 5 shows high-resolution BF and HAADF STEM images of AlN growth on a graphene island that remained on the etched surface of the $\mathrm{SiC}$, suggesting that the etching process was not completely perfect. From the cross-sectional images, the graphene island dimensions are estimated to be between 40 and $100 \mathrm{~nm}$. Bright
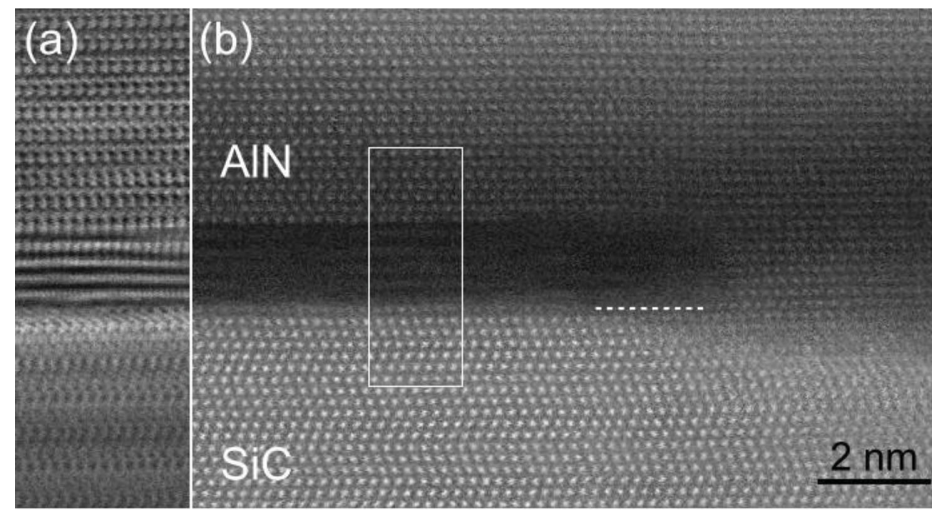

(c)

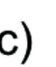

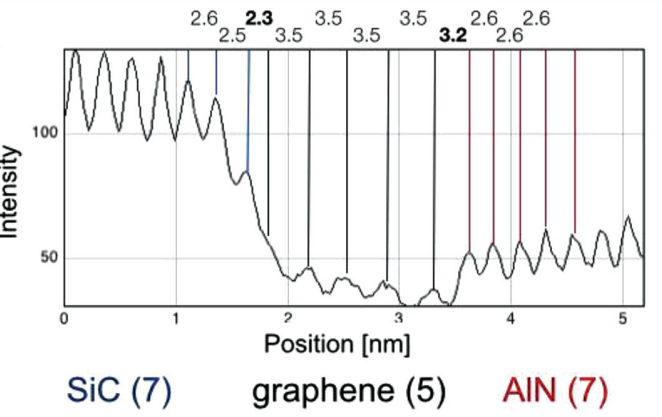

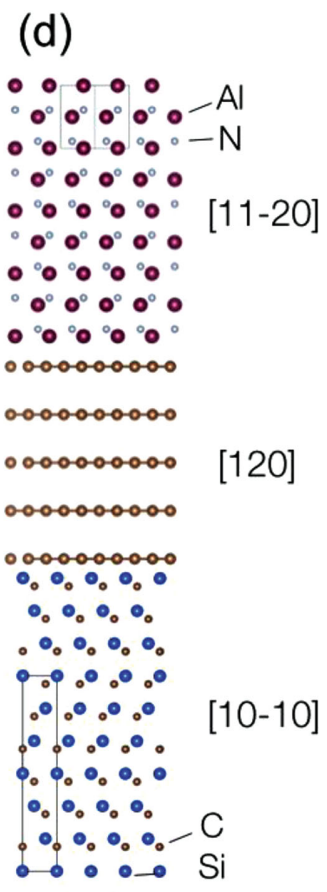

Figure 5. High-resolution aberration-corrected a) BF STEM and b) HAADF STEM images of an etched graphene/AIN interface. Dashed line marks the uppermost Si-C layer. The inner ADF detector semiangle used was $78 \mathrm{mrad}$. b) Integrated intensity scan across the interface with peak-to-peak distances recorded from the region marked by rectangle in (b). c) Proposed atomistic model of the interface deduced from the STEM images shown in (a, b). 


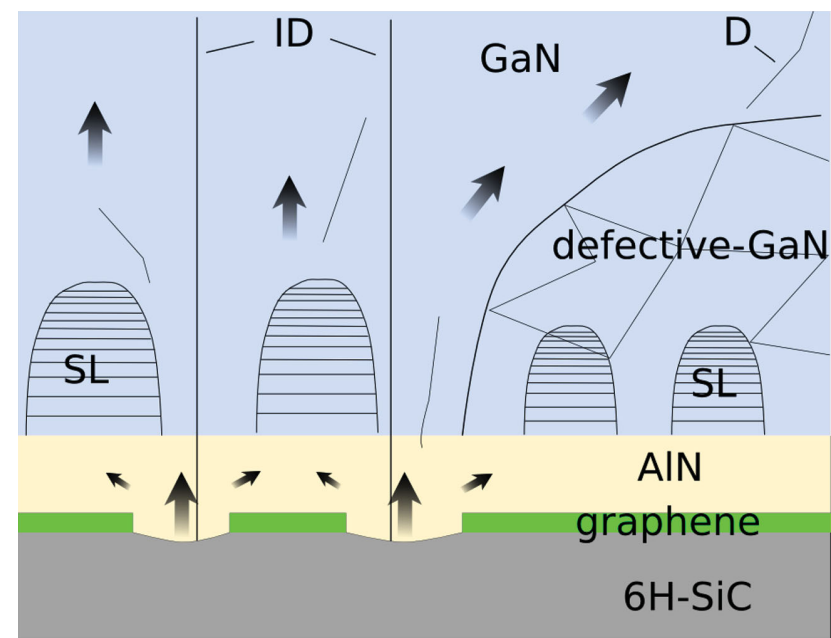

Figure 6. Growth of the nitride heterostructure on etched graphene layers inferred from the present experimental results. SL: GaN/AIN superlattice; ID: inversion domain; D: dislocation. Arrows indicate the growth direction.

spots in Figure 5b are likely to correspond to atomic columns of $\mathrm{Si}$ and $\mathrm{Al}$ in the $6 \mathrm{H}-\mathrm{SiC}$ and $\mathrm{AlN}$ layers, respectively. Based on the contrast, five carbon layers are thought to be present. On the right side of the image, the graphene layers have been etched away completely and the AlN grains have nucleated directly on the $6 \mathrm{H}-\mathrm{SiC}$. The white-dashed line in Figure $5 \mathrm{~b}$ indicates the uppermost $\mathrm{SiC}$ layer. The AlN grain starts to grow below the dashed line, indicating that the etching process also removed $\approx 1 \mathrm{~nm}$ of the $6 \mathrm{H}-\mathrm{SiC}$. The image suggests that the AlN nucleated and started to grow on the $6 \mathrm{H}-\mathrm{SiC}$ and then overgrew the graphene layers. Figure $5 \mathrm{c}$ shows integrated intensity lines scan obtained across the $\mathrm{SiC}$, graphene and $\mathrm{AlN}$, with the welldefined peaks corresponding to $\mathrm{Si}, \mathrm{C}$, and Al layers. Peak-topeak distances of $2.6 \pm 0.1 \AA$ for the $6 \mathrm{H}-\mathrm{SiC}$ and $\mathrm{AlN}$ layers match well with values from the literature for 0002 lattice plane distances of $2.51 \AA$ for $6 \mathrm{H}-\mathrm{SiC}$ and $2.59 \AA$ for AlN. For the graphene layers, the measured distance between the peaks is $3.5 \pm$ $0.1 \AA$, which is slightly higher than the expected value of $3.35 \AA$ for graphite. The first carbon layer grown on $\mathrm{SiC}$ is the strongly bound buffer layer ${ }^{[14-17]}$ at the interface that is separated from the uppermost $\mathrm{SiC}$ by $2.3 \AA$. This layer separation matches with values measured by other groups for graphene grown on $4 \mathrm{H}-$ $\mathrm{SiC}(0001)^{[18]}$ and $6 \mathrm{H}-\mathrm{SiC}(0001) .{ }^{[19]}$ Figure $5 \mathrm{~b}, \mathrm{c}$ also show that the intensity of the uppermost $\mathrm{Si}-\mathrm{C}$ layer is lower and its structure is less defined than that of Si columns in the $6 \mathrm{H}-\mathrm{SiC}$, suggesting a nonstoichiometric composition. The AlN grain and graphene are separated by a peak-to-peak separation of $\approx 3.2 \AA$ in Figure 5a. Figure 5c shows an atomic model of the AlN/graphene/Si-C/6H-SiC structure deduced from the high-resolution BF and HAADF STEM images.

\section{Summary and Conclusions}

Figure 6 shows a summary of the nitride heterostructure growth on graphene layers inferred from the present TEM results. $\mathrm{Ar} / \mathrm{O}_{2}$ plasma etching creates graphene islands on the
6H-SiC substrate and removes the uppermost monolayers of SiC. The AlN nucleates epitaxially on the SiC. The AlN above the graphene layers has the same orientation as the epitaxial AlN on $\mathrm{SiC}$, suggesting that AlN overgrows graphene islands laterally. In the control sample grown without graphene layers, the AlN buffer layer and $\mathrm{Al}_{0.2} \mathrm{Ga}_{0.8} \mathrm{~N}$ transition layer were clearly separated and created a sharp interface (see Supporting Information). In contrast to the control sample, the AlN surface on graphene is rough and an $\mathrm{AlN} / \mathrm{GaN}$ superlattice forms in the $\mathrm{Al}_{0.2} \mathrm{Ga}_{0.8} \mathrm{~N}$ transition layers, perhaps as a result of the presence of a small amount of carbon contamination originating from the graphene layers. It is striking that the $\mathrm{GaN}$ layer is defective above the intact graphene layers, with a crystallographic orientation that is different from that of the single crystalline $\mathrm{GaN}$ above the graphene islands. Inversion domains are found above the etched regions, where the nitride layers grow on $\mathrm{SiC}$. The single-crystalline $\mathrm{GaN}$ overgrows the polycrystalline $\mathrm{GaN}$ by self-assisted lateral epitaxial overgrowth, reducing the dislocation density and leading to a smooth $\mathrm{GaN}$ surface.

We have demonstrated the growth of high-quality $\mathrm{GaN}$ layers on etched graphene using an AlN buffer layer and an $\mathrm{Al}_{0.2} \mathrm{Ga}_{0.8} \mathrm{~N}$ transition layer. The use of plasma etching of graphene layers in the form of regular stripes leaves islands of graphene, next to which AlN grains nucleate by standard epitaxy on SiC. Polycrystalline GaN forms above the intact graphene layers, which act as a buffer for self-assisted epitaxial lateral overgrowth of a single-crystalline $\mathrm{GaN}$ layer. The resulting lateral growth reduces the defect density in the GaN. Inversion domains are found in the regions that form directly on the SiC. The probable presence of $\mathrm{C}$ contamination during growth of the $\mathrm{Al}_{0.2} \mathrm{Ga}_{0.8} \mathrm{~N}$ transition layer results in the growth of a phaseseparated AlN/GaN multilayer, which is not understood in detail at present.

Our results clearly show that nitride heterostructures deposited on patterned graphene layers are grown via lateral overgrowth, which results in the formation of a single-crystalline GaN layer that has a relatively low defect density and a smooth surface. Optimization of the plasma etching process and growth procedure promises to offer a simple route to produce nitridebased device structures with better thermal management due to graphene layer incorporation.

\section{Experimental Section}

TEM Specimen Preparation: $A \approx 15 \mu \mathrm{m}$ long thin section was cut out using a dual-beam focused ion beam (FIB) system using ion energies of 30 and $5 \mathrm{keV}$. A Pt/C protective layer was deposited onto the surface of the $\mathrm{GaN}$ using the electron and ion beams with thicknesses of 0.5 and $2.5 \mu \mathrm{m}$, respectively. The FIB lamella was fixed onto a standard $\mathrm{Cu}$ Omniprobe grid using $\mathrm{Pt} / \mathrm{C}$ layer deposition. The ion beam-induced surface damage was then reduced by using low-energy $(0.5 \mathrm{keV})$ focused Ar-ion milling at an incident angle of $10^{\circ}$ in a Fischione Nanomill 1040 system.

TEM Characterization: For conventional TEM studies, an FEI Tecnai G2 microscope was used at an accelerating voltage of $200 \mathrm{kV}$. For high-resolution STEM studies, a probe-aberration-corrected FEI Titan $80-300$ microscope was used at an accelerating voltage of $300 \mathrm{kV}$, with the aberration coefficients of the probe forming system corrected to fourth order. 


\section{Acknowledgements}

The Hungarian National Scientific Research Fund (OTKA) supported this work through Grant No. K108869. The authors acknowledge financial support from the European Union under the Seventh Framework Program under a contract for an Integrated Infrastructure Initiative. Reference 312483 - ESTEEM2. The authors would like to thank D. Meertens (Forschungszentrum Jülich, PGI-5) for the preparation of FIB lamellas. R.Y. acknowledges financial support from the Swedish NRC (VR).

Received: May 8, 2014

Revised: November 15, 2014

Published online:

[1] K. S. Novoselov, V. I. Falko, L. Colombo, P. R. Gellert, M. G. Schwab, K. A. Kim, Nature 2012, 490, 192.

[2] A. A. Balandin, Nat. Mater. 2011, 10, 569.

[3] R. J. Trew, D. S. Green, J. B. Shealy, IEEE Microwave Mag. 2009, 10, 116.

[4] M. H. Crawford, IEEE J. Sel. Top. Quantum Electron. 2009, 15, 1028.

[5] N. Han, T. V. Cuong, M. Han, B. D. Ryu, S. Chandramohan, J. B. Park, J. H. Kang, Y. J. Park, K. B. Ko, H. Y. Kim, J. H. Ryu, Y. S. Katharria, C. J. Choi, C. H. Hong, Nat. Commun. 2013, 4, 1452.

[6] K. Chung, K.C.-H. Lee, G. C. Yi, Science 2010, 330, 655.
www.MaterialsViews.com

[7] Z. Yan, G. Liu, J. M. Khan, A. A. Balandin, Nat. Commun. 2012, 3, 827.

[8] C. Virojanadara, M. Syväjarvi, A. A. Zakharov, T. Balasubramanian, R. Yakimova, L. I. Johansson, Phys. Rev. B 2008, 78, 245403.

[9] L. Kotis, S. Gurban, B. Pecz, M. Menyhard, R. Yakimova, Appl. Surf. Sci. 2014, 316, 301.

[10] J. S. Moon, D. Curtis, S Bui, M. Hu, D. K. Gaskill, J. L. Tedesco, P. Asbeck, G. G. Jernigan, B. L. VanMil, R. L. Myers-Ward, C. R. Eddy, P. M. Campbell, X. Weng, IEEE Electron Device Lett. 2010, 31, 260.

[11] P. L. Neumann, E. Tóvári, S. Csonka, K. Kamarás, Z. E. Horváth, L. P. Biró, Nucl. Instrum. Methods Phys. Res. B 2012, 282, 130.

[12] P. K. Mohseni, A. Behnan, J. D. Wood, C. D. English, J. W. Lyding, E. Pop, X. Li, Nano Lett. 2013, 13, 1153.

[13] Z. Yu, D. A. Muller, J. Silcox, J. Appl. Phys. 2010, 95, 3362.

[14] W. A. de Heer, C. Berger, M. Ruan, M. Sprinkle, X. Li, Y. Hu, B. Zhang, J. Hankinson, E. Conrad, Proc. Natl. Acad. Sci. USA 2011, $108,16900$.

[15] C. Virojanadara, R. Yakimova, A. A. Zakharov, L. I. Johansson, J. Phys. D: Appl. Phys. 2010, 43, 374010.

[16] K. V. Emtsev, F. Speck, T. Seyller, L. Ley, J. D. Riley, Phys. Rev B 2008, 77, 155303

[17] L. H. de Lima, A. de Siervo, R. Landers, G. A. Viana, Phys. Rev. B 2013, 87, $081403(\mathrm{R})$.

[18] J. Borysiuk, W. Strupiński, R. Bożek, A. Wysmołek, J. M. Baranowski, Mater. Sci. Forum 2009, 207, 615.

[19] W. Norimatsu, M. Kusunoki, Chem. Phys. Lett. 2009, 468, 54. 